\title{
Making myelin basic protein - from mRNA transport to localized translation
}

\author{
Christina Müller ${ }^{\dagger}$, Nina M. Bauer ${ }^{\dagger}$, Isabelle Schäfer and Robin White* \\ Institute of Physiology and Pathophysiology, University Medical Center of the Johannes Gutenberg University, Mainz, Germany
}

Edited by:

Martin Stangel, Hannover Medical

School, Germany

Reviewed by:

Matthias Klugmann, University of New South Wales, Australia

Hauke B. Werner, Max Planck Institute

of Experimental Medicine, Germany

\section{${ }^{*}$ Correspondence:}

Robin White, Institute of Physiology and Pathophysiology, University Medical Center of the Johannes Gutenberg University, Duesbergweg 6, 55128 Mainz, Germany e-mail:white@uni-mainz.de

${ }^{\dagger}$ Christina Müller and Nina M. Bauer have contributed equally to this work
In the central nervous system (CNS) of most vertebrates, oligodendrocytes enwrap neuronal axons with extensions of their plasma membrane to form the myelin sheath. Several proteins are characteristically found in myelin of which myelin basic protein (MBP) is the second most abundant one after proteolipid protein. The lack of functional MBP in rodents results in a severe hypomyelinated phenotype in the CNS demonstrating its importance for myelin synthesis. Mbp mRNA is transported from the nucleus to the plasma membrane and is translated locally at the axon-glial contact site. Axonal properties such as diameter or electrical activity influence the degree of myelination. As oligodendrocytes can myelinate many axonal segments with varying properties, localized MBP translation represents an important part of a rapid and axon-tailored synthesis machinery. MBP's ability to compact cellular membranes may be problematic for the integrity of intracellular membranous organelles and can also explain why MBP is transported in oligodendrocytes in the form of an mRNA rather than as a protein. Here we review the recent findings regarding intracellular transport and signaling mechanisms leading to localized translation of Mbp mRNA in oligodendrocytes. More detailed insights into the MBP synthesis pathway are important for a better understanding of the myelination process and may foster the development of remyelination therapies for demyelinating diseases.

\footnotetext{
Keywords: myelin basic protein, hnRNP A2, mRNA localization, oligodendrocyte, Fyn, RNA granule, translational regulation, review
}

\section{MYELIN BASIC PROTEIN}

In the central nervous system (CNS) oligodendrocytes produce and maintain myelin. The insulating multilamellar myelin sheath improves neuronal communication by increasing impulse propagation velocity in neuronal axons while limiting energy requirements. It is also becoming more and more apparent that oligodendrocytes provide trophic support for neuronal axons which is essential for axonal survival and integrity (Funfschilling et al., 2012; Lee et al., 2012). A lack or disruption of intact myelin is associated with several neurological disorders including multiple sclerosis and inherited leukodystrophies.

One of the major components of CNS myelin is myelin basic protein (MBP; Jahn et al., 2009), which has been referred to as the "executive molecule of myelin" (Boggs, 2006). Shiverer (shi) mice as well as long evans shaker (les) rats both lack functional MBP and are characterized by severe hypomyelination in the CNS, shivering symptoms, and premature death (Readhead and Hood, 1990; Kwiecien et al., 1998). Interestingly, compact myelin can be formed in the peripheral nervous system of both mutant rodents which seems to depend on a compensatory function of the P0 protein which is not expressed in the CNS (Martini etal., 1995). The deficiency of other myelin proteins such as proteolipid protein (PLP) and $2^{\prime}, 3^{\prime}$-cyclic nucleotide $3^{\prime}$-phosphodiesterase (CNP) seems to cause secondary neuronal effects rather than affecting CNS myelination as such (Klugmann et al., 1997; Griffiths et al., 1998; Lappe-Siefke et al., 2003).
The multifunctional classic MBP proteins arise from a gene complex called Golli (genes of oligodendrocyte lineage) which also gives rise to the Golli (-MBP) family of proteins (Campagnoni et al., 1993). The Golli gene has three different transcriptional start sites allowing the expression of the two distinct subfamilies of proteins, which are temporally and locally regulated. Whereas the presence of classic MBP proteins is mainly restricted to myelinating cells, Golli (-MBP) proteins have been described in other neural and non-neural cells (Fulton et al., 2010). The different MBP isoforms in the mouse $(14,17.22,17.24,18.5,20.2$, and $21.5 \mathrm{kDa}$ ) mainly stem from transcription start site 3 and are formed by differential splicing (Harauz and Boggs, 2013). All classic MBP isoforms are encoded by exons I, III, IV, and VII, while exon II, V, and VI are only found in specific splice variants.

Interestingly, different isoforms are developmentally regulated and have different cellular distributions. Exon II-containing isoforms $(17.22,20.2$, and $21.5 \mathrm{kDa})$ are expressed at high levels in early development, are spread throughout the cytoplasm and also accumulate in the nucleus (Allinquant etal., 1991; Smith et al., 2013). Nuclear $21.5 \mathrm{kDa} \mathrm{MBP}$ appears to influence the proliferation of immortalized N19 oligodendroglial cells and stimulates morphological changes in co-cultured neuronal N2a cells (Smith et al., 2013). Exon II-containing MBPs have also been found in compact myelin but appear to be enriched in the radial component of myelin (Karthigasan et al., 1996).

MBP Isoforms lacking exon II are located at the plasma membrane (Allinquant et al., 1991). Due to its positive charge MBP 
associates with the negatively charged oligodendroglial phospholipids and has traditionally been proposed to function primarily in the compaction of myelin membranes. It was shown in mice and zebrafish that phosphatidylinositol 4,5-bisphosphate (PIP2) recruits $\mathrm{MBP}$ to the plasma membrane and that this interaction can be counteracted by elevated calcium levels (Nawaz et al., 2009, 2013). MBPs function in myelin compaction by membrane association is obviously very important, but additional functions have been assigned to this molecule and may explain the drastic phenotypes observed in its absence.

It was recently shown that MBP protein is involved in regulating the protein to lipid ratio of myelin membranes by acting as a molecular sieve and by inhibiting the diffusion of membrane proteins with large cytosolic domains into myelin membrane sheets (Aggarwal et al., 2011). In these developing membrane sheets, MBP seems to oligomerize into a cohesive protein meshwork which drives other myelin proteins such as myelin-associated glycoprotein (MAG) or CNP out of the sheet to form a lipid rich insulating myelin membrane with only few remaining proteins, largely PLP and MBP (Aggarwal et al., 2013).

It has also been demonstrated that MBP interacts with cytoskeletal proteins and influences their bundling and polymerization (Dyer et al., 1994; Hill and Harauz, 2005; Hill et al., 2005).

In addition to these structural tasks, MBP has been connected to signaling pathways. MBP has the ability to bind signaling molecules such as Fyn kinase which is important for morphological differentiation and myelination (Kramer-Albers and White, 2011; Smith et al., 2012). Moreover, MBP binding to the plasma membrane modulates voltage-operated $\mathrm{Ca}^{2+}$ channels (VOCCs) and thereby affects $\mathrm{Ca}^{2+}$ responses in the cell (Smith et al., 2011).

The distinct functions as well as their regulation by posttranslational modifications are reviewed in detail elsewhere (Boggs, 2006; Harauz et al., 2009; Harauz and Boggs, 2013) and emphasize that MBP is an essential protein for many aspects of oligodendrocyte homeostasis and myelin formation. Here we review the synthesis of $\mathrm{MBP}$ and focus on the posttranscriptional events including mRNA transport and localized translation.

\section{Mbp mRNA IS LOCALIZED IN RNA TRANSPORT GRANULES}

As $M b p$ mRNA and ribosomes were found to be present in biochemically purified myelin fractions thirty years ago, it was postulated that $M b p$ mRNA is transported to the myelin compartment where translation occurs locally (Colman et al., 1982). This study also demonstrated the efficiency of this localization system. It was shown that newly synthesized MBP protein can be detected in myelin fractions as early as 2 min after translation in the actively myelinating brainstem of young rats. Following this, microinjection experiments with labeled $M b p$ mRNA revealed the formation of RNA transport granules which are moved on microtubules throughout the cytoplasm to the distal parts of oligodendrocyte processes (Ainger et al., 1993; Carson et al., 1997).

Cytoplasmic mRNA localization has been described for many mRNAs and cell types (Shahbabian and Chartrand, 2012). Commonly, different RNA binding proteins referred to as trans-acting factors bind to specific nucleotide sequences in the mRNA's 3'untranslated region (UTR) termed cis-acting factors or elements. mRNA localization can expand the control of cellular gene expression in a spatio-temporal manner by inhibiting mRNA translation until a specific location is reached at a defined time point.

The formation of RNA granules including a specific selection of the transported cargo needs to be tightly controlled. Members of the QKI family of proteins have been reported to influence nucleocytoplasmic transport as well as stabilization of Mbp mRNA ( $\mathrm{Li}$ et al., 2000; Larocque et al., 2002; Bockbrader and Feng, 2008; Wang et al., 2010).

Cytoplasmic transport of $M b p$ mRNA largely depends on an RNA binding protein termed heterogeneous nuclear ribonucleoprotein (hnRNP) A2 which binds to a specific sequence in the 3'UTR (Hoek et al., 1998). This sequence was initially termed the RNA transport signal (RTS) consisting of 21 nucleotides (Ainger et al., 1997) and it was subsequently shown that hnRNP A2 binding requires only 11 nucleotides (GCCAAGGAGCC) which are referred to as the A2 response element (A2RE; Munro et al., 1999). This cis-acting A2RE has been identified in several other mRNAs including glial myelin-associated oligodendrocytic basic protein (Mobp), Tau, carbonic anhydrase II (CaII), amyloid precursor protein $(A p p)$, as well as neuronal calcium/calmodulin-dependent protein kinase II $\alpha$ (CamKII $\alpha$ ), Neurogranin, and activity-regulated cytoskeleton-associated protein (Arc; Barbarese et al., 1999; Gao et al., 2008).

Alternative splicing of pre-mRNA encoded by the HnRNP $A 2 / B 1 / B 0$ gene results in the synthesis of the four isoforms hnRNP B1, A2, B1b, and A2b (Hatfield et al., 2002). In most of the studies dealing with hnRNP A2 and Mbp mRNA, antibodies were used which do not distinguish between the four isoforms in immunocytochemical experiments. Hence the drawn conclusions focused on the most abundant hnRNP A2 protein. It was recently suggested that hnRNP A2b is the predominant isoform in the cytoplasm of neural cells and that $M b p$ mRNA granule formation seems to depend on hnRNP A2b (Han et al., 2010). It may be the case that the described cytoplasmic functions of hnRNP A2 in the context of $M b p$ mRNA localization which we review here should be (mainly) attributed to hnRNP A2b and be taken into account when we refer to hnRNP A2.

The A2RE is encoded as part of exon VII and is hence present in every $M b p$ splice variant. Therefore, all of the $M b p$ mRNAs can potentially interact with hnRNP A2 and RNA granules. Interestingly, it was proposed that similar to the protein, also exon II-containing mRNAs are differentially localized in oligodendrocytes. It seems that exon II-containing Mbp mRNAs are located in the cell body whereas the exon II-lacking mRNAs are transported into the distal cellular processes (de Vries et al., 1997). It remains unclear how the exon II sequence influences the subcellular distribution of $M b p$ mRNAs encoding these 17.22, 20.2, and $21.5 \mathrm{kDa}$ isoforms.

In addition to the A2RE, Mbp mRNA contains an additional cis-acting sequence in its $3^{\prime}$ UTR termed the RNA localization region (RLR) (Ainger et al., 1997) or RNA localization signal (RLS; Barbarese et al., 1999). It has been proposed that the secondary structure of this sequence is required for specific localization of $M b p$ mRNA into the myelin compartment. 
Recently, additional RNA binding proteins were identified in $M b p$ mRNA granules (Raju et al., 2008; Laursen et al., 2011; White etal., 2012). HnRNP $\mathrm{K}$ as well as hnRNP $\mathrm{F}$ are both associated with Mbp mRNA and hnRNP A2 and influence the synthesis of MBP protein (Laursen etal., 2011; White et al., 2012). Coimmunoprecipitation of hnRNP F with hnRNP A2 is RNAse resistant while the copurification of hnRNP A2 with hnRNP K seems to depend on RNA. Thus hnRNP F is possibly recruited to the RNA granule by hnRNP A2 while hnRNP $\mathrm{K}$ binds to $M b p$ mRNA directly to a yet undefined region. The knock down of hnRNP $\mathrm{K}$ appears to abolish the transport of $M b p$ mRNA from process branch points to the most distal parts of oligodendrocyte processes and suggests a role of hnRNP $\mathrm{K}$ during this part of the transport path (Laursen etal., 2011). Earlier studies had already observed oligodendroglial hnRNP $\mathrm{K}$ in granular structures in the more proximal and not the distal parts of the processes (Kosturko et al., 2005), but it seems that the cellular distribution of hnRNP $\mathrm{K}$ depends on the differentiation status of the oligodendrocyte (Laursen et al., 2011).

In addition to hnRNP $\mathrm{K}$ and hnRNP $\mathrm{F}$, the A/B type hnRNP CBF-A was identified in $M b p$ mRNA granules. CBF-A binds to the RTS of $M b p$ mRNA, coimmunoprecipitates with hnRNP A2, $\mathrm{A} 3$ as well as $\mathrm{U}$ and knock down of CBF-A in the immortalized oligodendrocyte precursor cell (OPC) line Oli-neu inhibits the transport of $M b p$ mRNA to the processes (Raju et al., 2008). It appears that hnRNP K and CBF-A are important for different parts of the transport path from the cytosol to the most distal regions of the processes, but this needs to be addressed in more detail in the future.

HnRNP F is tyrosine-phosphorylated by Fyn kinase (White etal., 2012) and hnRNP $K$ appears to become tyrosinephosphorylated in oligodendrocytes cultured on laminin for 4 days (Laursen et al., 2011). Although it has been shown that laminin stimulates oligodendroglial Fyn activity and that Fyn interacts with hnRNP K in the CNS (Kai et al., 1997), it remains to be shown if hnRNP $\mathrm{K}$ is a target of Fyn. The influence of Fyn activation on translation of MBP is discussed below.

It was postulated that Mbp mRNA is transported on microtubules to the myelin compartment and that this transport requires kinesin as the translocation of microinjected $M b p$ mRNA to the myelin sheets of cultured oligodendrocytes is perturbed by drugs affecting microtubule dynamics or by kinesin antisense RNA treatment (Carson et al., 1997). Furthermore, oligodendrocytes derived from the hypomyelinated taiep rat mutant have abnormally accumulated microtubules and show restricted transport of Mbp mRNA granules (Song et al., 2003). As shown in zebrafish, the kinesin motorprotein Kiflb is required for the transport of $M b p$ mRNA toward the processes of myelinating oligodendrocytes and Kif1b mutants show ectopic localization of MBP protein as well as misplaced myelin like membranes (Lyons et al., 2009). The microtubule associated protein tumor overexpressed gene (TOG) colocalizes with $M b p$ mRNA and hnRNP A2 in granules and might be involved in the regulation of kinesin activity, thereby directing granule transport toward the plus end of the microtubule (Kosturko et al., 2005).

\section{TRANSLATIONAL REPRESSION DURING Mbp mRNA TRANSPORT}

It has been proposed that RNA granules contain all necessary molecules for the translation of mRNA and indeed a number of relevant molecules have been identified in $M b p$ mRNA granules. These include arginyl-tRNA synthetase (ATS), elongation factor 1a (EF1a), and ribosomal RNA (Barbarese et al., 1995). If mRNA granules are more or less ready to translate the transported mRNAs, then premature or ectopic translation must be avoided until certain signals set off the protein synthesis machinery. Regarding $M b p$ mRNA, hnRNP E1 and the small non-coding RNA 715 (sncRNA715) have been directly connected to translational inhibition during transport (Kosturko et al., 2006; Bauer et al., 2012), while the function of other granule molecules such as hnRNP F, TOG, and hnRNP $\mathrm{K}$ in respect to translational control may have a more indirect effect on the MBP synthesis path (Francone et al., 2007; Laursen et al., 2011; White et al., 2012).

HnRNP E1 partially colocalizes with hnRNP A2 and microinjected A2RE-containing mRNA in oligodendrocytes (Kosturko et al., 2006). Furthermore hnRNP E1 inhibits the translation of an A2RE-containing Green fluorescent protein reporter mRNA in microinjected B104 neuroblastoma cells and in a rabbit reticulocyte lysate-based in vitro assay (Kosturko et al., 2006). Additional experiments lead to the conclusion that hnRNP E1 is recruited to RNA granules by hnRNP A2 and inhibits the translation of A2RE-containing mRNAs during transport (Kosturko et al., 2006).

In the light of the emerging importance of post-transcriptional gene regulation by small RNA molecules such as microRNAs or endogenous siRNAs, translational inhibition of localized mRNAs by these types of molecules appears to be a suitable mechanism and has been reported in neurons (McNeill and Van Vactor, 2012). Recently, sncRNA715 was revealed to inhibit the synthesis of endogenous MBP in primary oligodendrocytes via binding to a specific recognition site in the $3^{\prime}$ UTR of $M b p$ mRNA (Bauer et al., 2012). SncRNA715 copurifies with hnRNP A2 and Mbp mRNA biochemically and is located in granular structures in the cytoplasm and processes of cultured oligodendrocytes. Interestingly, the analysis of chronic multiple sclerosis lesions containing OPCs and $M b p$ mRNA but lacking MBP protein revealed abnormally high levels of sncRNA715 compared to normal appearing white matter (NAWM; Bauer et al., 2012). These data not only allude to an evolutionary conserved mechanism of MBP translational regulation, but may also contribute to a better understanding of why OPCs fail to differentiate appropriately to remyelinate axons in late stages of MS.

While yet unclear for hnRNP E1, sncRNA715 seems to regulate all MBP isoforms as the binding sequence is present in all of the Mbp mRNA 3'UTRs and Western blot analysis revealed a translational repression of every isoform (Bauer et al., 2012). So far it is unknown if hnRNP E1 and sncRNA715 synergistically influence $\mathrm{MBP}$ translation in oligodendrocytes or if they act at different time points or cellular locations.

Conceptually, one could distinguish two aspects of translational repression of $M b p$ mRNA. As $M b p$ mRNA can be detected in OPCs which do not yet synthesize MBP protein, MBP translation may generally be repressed in OPCs until a certain degree of differentiation has been reached at which defined (axonal) signals initiate 
MBP protein synthesis. In myelinating oligodendrocytes MBP protein synthesis may be repressed during intracellular transport to prevent ectopic localization of MBP which may have deleterious consequences by compacting intracellular membranes (Staugaitis et al., 1990). The latter also appears to be very important for a specific synthesis machinery capable of producing defined amounts of myelin in response to axonal determinants such as axon diameter or activity.

A number of molecules participate in the transport of $M b p$ mRNA in oligodendrocytes and are proposed to function in RNA granule formation, transport and translational inhibition (summarized in Table $\mathbf{1}$ and Figure 1). RNA deep sequencing and advanced proteomics will most likely identify additional regulatory molecules in the future.

\section{TRANSLATIONAL DE-REPRESSION AND LOCAL MBP SYNTHESIS}

Translationally inhibited mRNAs in RNA granules have been shown to be de-repressed in order to initiate protein synthesis at a specific time point or localization in cells (Ostareck-Lederer et al., 2002; Huttelmaier et al., 2005).

As mentioned above oligodendrocytes must regulate the amount of myelin that is produced at specific axonal segments and as these amounts may vary, it seems to be the case that at least parts of the myelin synthesis machinery is decentralized to respond to axonal requirements locally. Src-family non-receptor tyrosine kinases have been implicated with the initiation of localized translation of transported mRNAs by phosphorylation of trans-acting factors (Ostareck-Lederer et al., 2002; Huttelmaier et al., 2005).

In oligodendrocytes Fyn kinase is the predominant Src-family kinase and is an important regulator of myelination (KramerAlbers and White, 2011). In a screen for Fyn substrates in oligodendroglial cells, hnRNP A2 was identified and an axonalglial signaling pathway was suggested controlling the activation of Fyn in order to trigger MBP translation at the axon-glial contact site (White et al., 2008). It was shown that binding of the axonal cell adhesion molecule L1 to oligodendroglial F3/Contactin activates Fyn kinase which phosphorylates hnRNP A2 and promotes translation of A2RE-containing mRNAs (White et al., 2008).

It was later contributed that F3/Contactin forms a complex with $\alpha 6 \beta 1$ integrins and that L1 binding enhances myelination

Table 1 | Molecules associated with MBP mRNA during cytoplasmic localization as mentioned in the text.

\begin{tabular}{lll}
\hline Molecule & Binding region & Reference \\
\hline hnRNP A2 & A2RE within RTS in 3'UTR & Hoek et al. (1998) \\
hnRNP K & Undefined & Laursen et al. (2011) \\
hnRNP F & Presumably via hnRNP A2 & White et al. (2012) \\
hnRNP CBF-A & RTS in 3'UTR & Raju et al. (2008) \\
TOG & Presumably via hnRNP A2 & Kosturko et al. (2005) \\
sncRNA 715 & Specific recognition site in 3'UTR & Bauer et al. (2012) \\
hnRNP E1 & Presumably via hnRNP A2 & Kosturko et al. (2006)
\end{tabular}

in a co-culture system which can be blocked by the addition of antibodies directed against $\beta 1$ integrins (Laursen et al., 2009), suggesting a coordinated regulation of Fyn activation and myelination by the extracellular matrix and the axonal surface.

The stimulation of $\alpha 6 \beta 1$ integrins by laminin binding increases Fyn activity (Laursen et al., 2009) and the presence of a constitutively active $\beta 1$ integrin mutant promotes MBP translation (Laursen etal., 2011). As described above the granule protein hnRNP F is phosphorylated by Fyn. Active Fyn releases hnRNP $F$ from the granule and reduces the amount of $M b p$ mRNA bound to hnRNP F (White etal., 2012). As hnRNP A2 and hnRNP E1 also appear to be released from the granule in these conditions (White etal., 2008) it seems that Fyn activation results in a breakdown of the granule by phosphorylation of granule proteins, releasing $M b p$ mRNA from its inhibitors at the axonglial contact site and allowing localized MBP protein synthesis to occur. It may be the case that sncRNA715 is also separated from $M b p$ mRNA in response to Fyn activity by a yet unknown mechanism.

Interestingly, it was recently shown that electrical stimulation increases the axonal surface expression of L1 as well as Fyn activation, local MBP synthesis and myelination all of which appears to be regulated by axonal vesicular glutamate release (Wake et al., 2011).

All of the above mentioned studies emphasize the central role of Fyn kinase in translational regulation of MBP. This has strong implications for the myelination process, as demonstrated in vivo, as mice are hypomyelinated in the CNS in the absence of Fyn or in the presence of mutated inactive Fyn (Sperber et al., 2001). Moreover, MBP levels are reduced in Fyn knockout mice and Fyn phosphorylates QKI proteins modulating binding and stabilization of Mbp mRNAs (Zhang et al., 2003; Lu et al., 2005). In an elegant recent in vivo myelination study in zebrafish it was shown that Fyn regulates the number of myelin sheaths per oligodendrocyte (Czopka et al., 2013).

Intriguingly, active Fyn binds to $\alpha$-Tubulin as well as the microtubule associated protein Tau in oligodendrocytes and it was proposed that activated Fyn recruits the cytoskeleton toward the axon-glial contact site (Klein et al., 2002). As mentioned above, $M b p$ mRNA granules are transported on microtubules toward the periphery of the cell, so that activation of Fyn could recruit $M b p$ mRNA to the axon-glial contact site to initiate the myelination process by synthesizing MBP. However, it is likely that also Fynindependent pathways regulate the translation of $M b p$ mRNA. Interestingly, a function of TOG in the synthesis of MBP has been suggested which does not seem to be dependent on granule transport (Francone et al., 2007). Nevertheless, a lot of data provide evidence for an intercellular signaling pathway originating from active neuronal axons which recruits $M b p$ mRNA and induces localized MBP synthesis and myelination.

\section{CONCLUSION}

The overall synthesis of MBP is a complex and highly regulated mechanism and we have only discussed elements of it here. As summarized in Figure 1, Mbp mRNA is transported in large ribonucleoprotein complexes on microtubules toward the oligodendroglial plasma membrane. A number of proteins and RNAs 


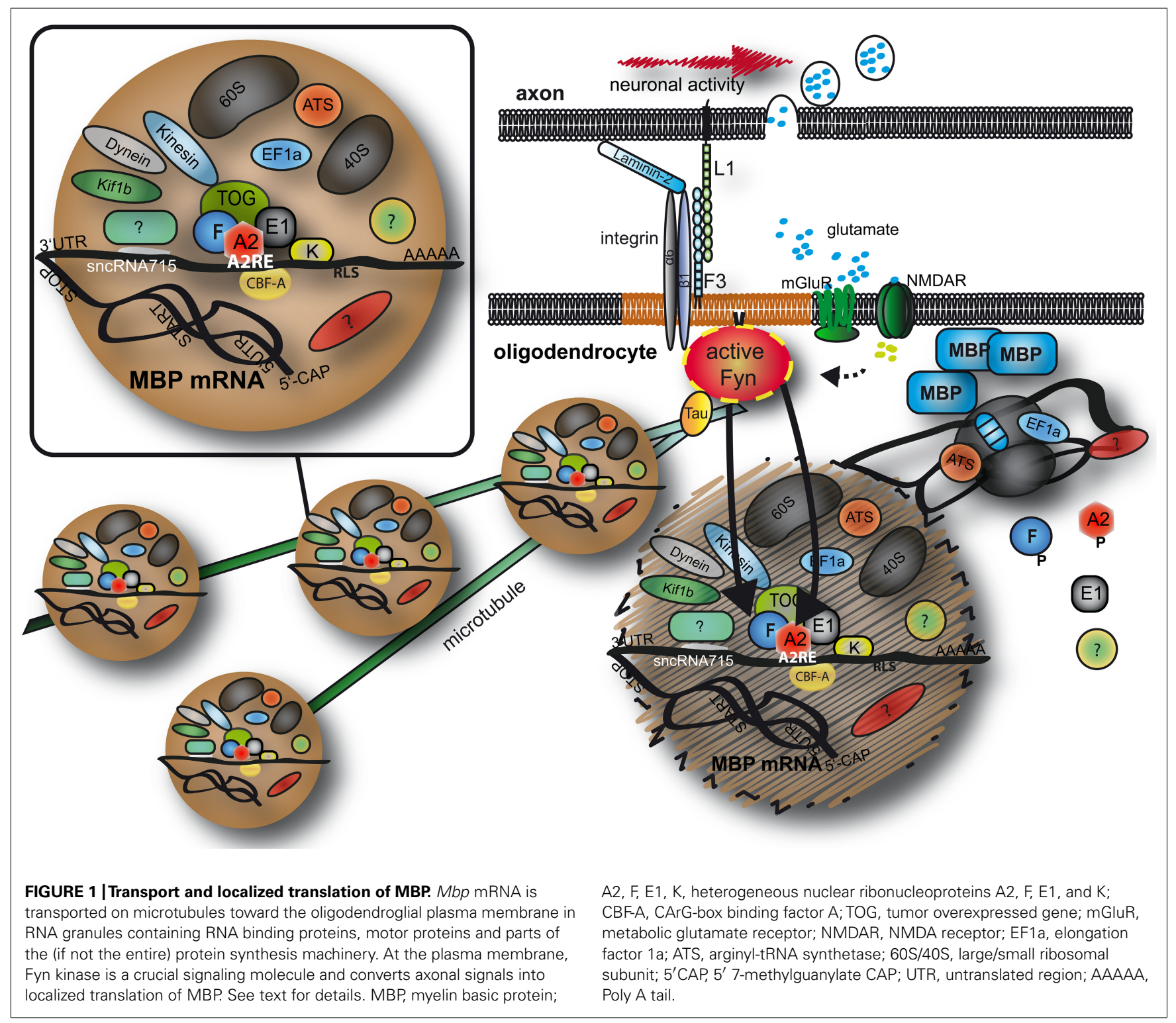

form these granules and MBP translation is repressed during transport. It seems that axon-glial signaling events recruit the granules and stimulate localized translation of MBP allowing axon-tailored myelination to ensue. It is likely that RNA transport granules are highly dynamic and potentially change their composition during intracellular transport so that a critical function of individual molecules is restricted to individual parts of the localization event. Future investigations will help to improve our understanding of the regulation and composition of heterogeneous granules which will have important implications for the myelination procedure.

\section{REFERENCES}

Aggarwal, S., Snaidero, N., Pahler, G., Frey, S., Sanchez, P., Zweckstetter, M., et al. (2013). Myelin membrane assembly is driven by a phase transition of myelin basic proteins into a cohesive protein meshwork. PLoS Biol. 11:e1001577. doi: 10.1371/journal. pbio. 1001577

Aggarwal, S., Yurlova, L., Snaidero, N., Reetz, C., Frey, S., Zimmermann,

\section{ACKNOWLEDGMENTS}

This work was supported by scholarships of the Focus Program Translational Neuroscience Mainz (Christina Müller) and Stipendienstiftung Rheinland-Pfalz (Nina M. Bauer) and by grants by the MAIFOR program (Mainz) and Deutsche Forschungsgemeinschaft (DFG WH168/1-1) (to Robin White). We thank Heiko Luhmann for ongoing support and Jacqueline Trotter for critical comments on the manuscript. We sincerely apologize for not being able to mention and cite all of the excellent work that has contributed to this exciting field of research.

J., et al. (2011). A size barrier limits protein diffusion at the cell surface to generate lipid-rich myelinmembrane sheets. Dev. Cell 21, 445-456. doi: 10.1016/j.devcel.2011. 08.001
Ainger, K., Avossa, D., Diana, A. S., Barry, C., Barbarese, E., and Carson, J. H. (1997). Transport and localization elements in myelin basic protein mRNA. J. Cell Biol. 138, 1077-1087. doi: $10.1083 /$ jcb.138.5.1077 
Ainger, K., Avossa, D., Morgan, F., Hill, S. J., Barry, C., Barbarese, E., et al. (1993). Transport and localization of exogenous myelin basic protein mRNA microinjected into oligodendrocytes. J. Cell Biol. 123, 431-441. doi: $10.1083 /$ jcb.123.2.431

Allinquant, B., Staugaitis, S. M., D’urso, D., and Colman, D. R. (1991). The ectopic expression of myelin basic protein isoforms in Shiverer oligodendrocytes: implications for myelinogenesis. J. Cell Biol. 113, 393 403. doi: 10.1083/jcb.113.2.393

Barbarese, E., Brumwell, C., Kwon, S., Cui, H., and Carson, J. H. (1999). RNA on the road to myelin. J. Neurocytol. 28, 263-270. doi: 10.1023/A:1007097226688

Barbarese, E., Koppel, D. E., Deutscher, M. P., Smith, C. L., Ainger, K., Morgan, F., et al. (1995). Protein translation components are colocalized in granules in oligodendrocytes. J. Cell Sci. 108(Pt 8), 2781-2790.

Bauer, N. M., Moos, C., Van Horssen, J., Witte, M., Van Der Valk, P., Altenhein, B., et al. (2012). Myelin basic protein synthesis is regulated by small non-coding RNA 715. EMBO Rep. 13, 827-834. doi: 10.1038/embor. 2012.97

Bockbrader, K., and Feng, Y. (2008). Essential function, sophisticated regulation and pathological impact of the selective RNA-binding protein QKI in CNS myelin development. Future Neurol. 3, 655-668. doi: 10.2217/14796708.3.6.655

Boggs, J. M. (2006). Myelin basic protein: a multifunctional protein. Cell. Mol. Life. Sci. 63, 1945-1961. doi: 10.1007/s00018-006-6094-7

Campagnoni, A. T., Pribyl, T. M., Campagnoni, C. W., Kampf, K., AmurUmarjee, S., Landry, C. F., et al. (1993). Structure and developmental regulation of Golli-mbp, a 105kilobase gene that encompasses the myelin basic protein gene and is expressed in cells in the oligodendrocyte lineage in the brain. J. Biol. Chem. 268, 4930-4938.

Carson, J. H., Worboys, K., Ainger, K., and Barbarese, E. (1997). Translocation of myelin basic protein mRNA in oligodendrocytes requires microtubules and kinesin. Cell Motil. Cytoskeleton 38, 318-328. doi: 10.1002/(SICI) 1097 0169(1997)38:4<318::AID-CM2>3. $0 . \mathrm{CO} ; 2-\#$

Colman, D. R., Kreibich, G., Frey, A. B., and Sabatini, D. D. (1982). Synthesis and incorporation of myelin polypeptides into CNS myelin. J. Cell Biol. 95, 598-608. doi: 10.1083/jcb.95.2.598
Czopka, T., Ffrench-Constant, C., and Lyons, D. A. (2013). Individual oligodendrocytes have only a few hours in which to generate new myelin sheaths in vivo. Dev. Cell 25, 599-609. doi: 10.1016/j.devcel.2013.05.013

de Vries, H., De Jonge, J. C., Schrage, C., Van Der Haar, M. E., and Hoekstra, D. (1997). Differential and cell development-dependent localization of myelin mRNAs in oligodendrocytes. J. Neurosci. Res. 47 479-488. doi: 10.1002/(SICI)10974547(19970301)47:5<479::AID-JNR 3>3.0.CO;2-E

Dyer, C. A., Philibotte, T. M., Wolf, M. K., and Billings-Gagliardi, S. (1994). Myelin basic protein mediates extracellular signals that regulate microtubule stability in oligodendrocyte membrane sheets. J. Neurosci. Res. 39 97-107. doi: 10.1002/jnr.490390112

Francone, V. P., Maggipinto, M. J., Kosturko, L. D., and Barbarese, E. (2007). The microtubule-associated protein tumor overexpressed gene/cytoskeleton-associated protein 5 is necessary for myelin basic protein expression in oligodendrocytes. J. Neurosci. 27, 7654-7662. doi: 10.1523/JNEUROSCI.0203-07.2007

Fulton, D., Paez, P. M., and Campagnoni, A. T. (2010). The multiple roles of myelin protein genes during the development of the oligodendrocyte. ASN Neuro 2, e00027. doi: 10.1042/AN20090051

Funfschilling, U., Supplie, L. M., Mahad, D., Boretius, S., Saab, A. S., Edgar, J., et al. (2012). Glycolytic oligodendrocytes maintain myelin and long-term axonal integrity. Nature 485, 517-521. doi: 10.1038/ nature 11007

Gao, Y., Tatavarty, V., Korza, G., Levin, M. K., and Carson, J. H. (2008). Multiplexed dendritic targeting of alpha calcium calmodulin-dependent protein kinase II, neurogranin, and activityregulated cytoskeleton-associated protein RNAs by the A2 pathway. Mol. Biol. Cell 19, 2311-2327. doi: 10.1091/mbc.E07-09-0914

Griffiths, I., Klugmann, M., Anderson, T., Yool, D., Thomson, C., Schwab, M. H., et al. (1998). Axonal swellings and degeneration in mice lacking the major proteolipid of myelin. Science $280,1610-1613$. doi: 10.1126/science.280.5369.1610

Han, S. P., Friend, L. R., Carson, J. H., Korza, G., Barbarese, E., Maggipinto, M., et al. (2010). Differential subcellular distributions and trafficking functions of hnRNP A2/B1 spliceoforms. Traffic 11, 886-898. doi: 10.1111/j.1600-0854.2010.01072.x
Harauz, G., and Boggs, J. M. (2013). Myelin management by the $18.5-\mathrm{kDa}$ and $21.5-\mathrm{kDa}$ classic myelin basic protein isoforms. J. Neurochem. 125, 334-361. doi: 10.1111/jnc.12195

Harauz, G., Ladizhansky, V., and Boggs, J. M. (2009). Structural polymorphism and multifunctionality of myelin basic protein. Biochemistry 48, 8094-8104. doi: 10.1021/bi901005f

Hatfield, J. T., Rothnagel, J. A., and Smith, R. (2002). Characterization of the mouse hnRNP A2/B1/B0 gene and identification of processed pseudogenes. Gene 295, 33-42. doi: 10.1016/S0378-1119(02)00800-4

Hill, C. M., and Harauz, G. (2005). Charge effects modulate actin assembly by classic myelin basic protein isoforms. Biochem. Biophys. Res. Commun. 329, 362-369. doi: 10.1016/j.bbrc.2005.01.151

Hill, C. M., Libich, D. S., and Harauz, G. (2005). Assembly of tubulin by classic myelin basic protein isoforms and regulation by post-translational modification. Biochemistry 44, 16672-16683. doi: 10.1021/bi050646+

Hoek, K. S., Kidd, G. J., Carson, J. H., and Smith, R. (1998). hnRNP A2 selectively binds the cytoplasmic transport sequence of myelin basic protein mRNA. Biochemistry 37, 7021-7029. doi: 10.1021/bi9800247

Huttelmaier, S., Zenklusen, D., Lederer, M., Dictenberg, J., Lorenz, M., Meng, X., et al. (2005). Spatial regulation of beta-actin translation by Src-dependent phosphorylation of ZBP1. Nature 438, 512-515. doi: 10.1038 /nature 04115

Jahn, O., Tenzer, S., and Werner, H. B. (2009). Myelin proteomics: molecular anatomy of an insulating sheath. Mol. Neurobiol. 40, 55-72. doi: 10.1007/s12035-009-8071-2

Kai, N., Mishina, M., and Yagi, T. (1997). Molecular cloning of Fyn-associated molecules in the mouse central nervous system. J. Neurosci. Res. 48 407-424. doi: 10.1002/(SICI)10974547(19970601)48:5<407::AID-JNR 3>3.0.CO;2-I

Karthigasan, J., Garvey, J. S., Ramamurthy, G. V., and Kirschner, D. A. (1996). Immunolocalization of 17 and $21.5 \mathrm{kDa}$ MBP isoforms in compact myelin and radial component. J. Neurocytol. 25, 1-7. doi: 10.1007/BF02284781

Klein, C., Kramer, E. M., Cardine, A. M., Schraven, B., Brandt, R., and Trotter, J. (2002). Process outgrowth of oligodendrocytes is promoted by interaction of fyn kinase with the cytoskeletal protein tau. J. Neurosci. 22, 698-707.
Klugmann, M., Schwab, M. H., Puhlhofer, A., Schneider, A., Zimmermann, F., Griffiths, I. R., et al. (1997). Assembly of CNS myelin in the absence of proteolipid protein. $\mathrm{Neu}$ ron 18, 59-70. doi: 10.1016/S08966273(01)80046-5

Kosturko, L. D., Maggipinto, M. J., D’sa, C., Carson, J. H., and Barbarese, E. (2005). The microtubule-associated protein tumor overexpressed gene binds to the RNA trafficking protein heterogeneous nuclear ribonucleoprotein A2. Mol. Biol. Cell 16, 1938-1947. doi: 10.1091/mbc.E0408-0709

Kosturko, L. D., Maggipinto, M. J., Korza, G., Lee, J. W., Carson, J. H., and Barbarese, E. (2006). Heterogeneous nuclear ribonucleoprotein (hnRNP) E1 binds to hnRNP A2 and inhibits translation of $\mathrm{A} 2$ response element mRNAs. Mol. Biol. Cell 17, 35213533. doi: 10.1091/mbc.E05-10-0946 Kramer-Albers, E. M., and White, R. (2011). From axon-glial signalling to myelination: the integrating role of oligodendroglial Fyn kinase. Cell. Mol. Life. Sci. 68, 2003-2012. doi: 10.1007/s00018-010-0616-z

Kwiecien, J. M., O'connor, L. T., Goetz, B. D., Delaney, K. H., Fletch, A. L., and Duncan, I. D. (1998). Morphological and morphometric studies of the dysmyelinating mutant, the Long Evans shaker rat. J. Neurocytol. 27, 581-591. doi: 10.1023/A:1006922227791

Lappe-Siefke, C., Goebbels, S., Gravel, M., Nicksch, E., Lee, J., Braun, P. E., et al. (2003). Disruption of Cnp1 uncouples oligodendroglial functions in axonal support and myelination. Nat. Genet. 33, 366374. doi: $10.1038 / n g 1095$

Larocque, D., Pilotte, J., Chen, T., Cloutier, F., Massie, B., Pedraza, L., et al. (2002). Nuclear retention of MBP mRNAs in the quaking viable mice. Neuron 36, 815-829. doi: 10.1016/S0896-6273(02)01055-3

Laursen, L. S., Chan, C. W., and FfrenchConstant, C. (2009). An integrincontactin complex regulates CNS myelination by differential Fyn phosphorylation. J. Neurosci. 29, 91749185. doi: 10.1523/JNEUROSCI. 5942-08.2009

Laursen, L. S., Chan, C. W., and Ffrench-Constant, C. (2011). Translation of myelin basic protein mRNA in oligodendrocytes is regulated by integrin activation and hnRNP-K. J. Cell Biol. 192, 797-811. doi: 10.1083/jcb.201007014

Lee, Y., Morrison, B. M., Li, Y., Lengacher, S., Farah, M. H., Hoffman, P. N., et al. (2012). Oligodendroglia metabolically support axons 
and contribute to neurodegeneration. Nature 487, 443-448. doi: 10.1038 /nature 11314

Li, Z., Zhang, Y., Li, D., and Feng, Y. (2000). Destabilization and mislocalization of myelin basic protein mRNAs in quaking dysmyelination lacking the QKI RNA-binding proteins. J. Neurosci. 20, 4944-4953.

Lu, Z., Ku, L., Chen, Y., and Feng, Y. (2005). Developmental abnormalities of myelin basic protein expression in fyn knock-out brain reveal a role of Fyn in posttranscriptional regulation. J. Biol. Chem. 280, 389-395. doi: 10.1074/jbc.M405973200

Lyons, D. A., Naylor, S. G., Scholze, A., and Talbot, W. S. (2009). Kiflb is essential for mRNA localization in oligodendrocytes and development of myelinated axons. Nat. Genet. 41, 854-858. doi: 10.1038/ng.376

Martini, R., Mohajeri, M. H., Kasper, S., Giese, K. P., and Schachner, M. (1995). Mice doubly deficient in the genes for $\mathrm{P} 0$ and myelin basic protein show that both proteins contribute to the formation of the major dense line in peripheral nerve myelin. $J$. Neurosci. 15, 4488-4495.

McNeill, E., and Van Vactor, D. (2012). MicroRNAs shape the neuronal landscape. Neuron 75, 363-379. doi: 10.1016/j.neuron.2012.07.005

Munro, T. P., Magee, R. J., Kidd, G. J., Carson, J. H., Barbarese, E., Smith, L. M., et al. (1999). Mutational analysis of a heterogeneous nuclear ribonucleoprotein A2 response element for RNA trafficking. J. Biol. Chem. 274, 34389-34395. doi: 10.1074/jbc.274.48.34389

Nawaz, S., Kippert, A., Saab, A. S., Werner, H. B., Lang, T., Nave, K. A., et al. (2009). Phosphatidylinositol 4,5-bisphosphate-dependent interaction of myelin basic protein with the plasma membrane in oligodendroglial cells and its rapid perturbation by elevated calcium.
J. Neurosci. 29, 4794-4807. doi: 10.1523/JNEUROSCI.3955-08.2009

Nawaz, S., Schweitzer, J., Jahn, O. and Werner, H. B. (2013). Molecular evolution of myelin basic protein, an abundant structural myelin component. Glia 61, 1364-1377. doi: 10.1002/glia.22520

Ostareck-Lederer, A., Ostareck, D. H., Cans, C., Neubauer, G., Bomsztyk, K., Superti-Furga, G., et al. (2002). c-Src-mediated phosphorylation of hnRNP K drives translational activation of specifically silenced mRNAs. Mol. Cell. Biol. 22, 4535-4543. doi: 10.1128/MCB.22.13.4535-4543. 2002

Raju, C. S., Goritz, C., Nord, Y. Hermanson, O., Lopez-Iglesias, C. Visa, N., etal. (2008). In cultured oligodendrocytes the A/B-type hnRNP CBF-A accompanies MBP mRNA bound to mRNA trafficking sequences. Mol. Biol. Cell 19, 30083019. doi: 10.1091/mbc.E07-10-1083 Readhead, C., and Hood, L. (1990). The dysmyelinating mouse mutations shiverer (shi) and myelin deficient (shimld). Behav. Genet. 20, 213-234. doi: 10.1007/BF01067791

Shahbabian, K., and Chartrand, P. (2012). Control of cytoplasmic mRNA localization. Cell. Mol. Life. Sci. 69, 535-552. doi: 10.1007 /s00018-011-0814-3

Smith, G. S., De Avila, M., Paez, P. M., Spreuer, V., Wills, M. K., Jones, N., et al. (2012). Proline substitutions and threonine pseudophosphorylation of the SH3 ligand of $18.5-\mathrm{kDa}$ myelin basic protein decrease its affinity for the Fyn-SH3 domain and alter process development and protein localization in oligodendrocytes. J. Neurosci. Res. 90, 28-47. doi: 10.1002/jnr.22733

Smith, G. S., Paez, P. M., Spreuer, V., Campagnoni, C. W., Boggs, J. M., Campagnoni, A. T., et al. (2011). Classical 18.5-and 21.5-kDa isoforms of myelin basic protein inhibit calcium influx into oligodendroglial cells, in contrast to golli isoforms J. Neurosci. Res. 89, 467-480. doi: 10.1002/jnr.22570

Smith, G. S., Samborska, B., Hawley, S. P., Klaiman, J. M., Gillis, T. E., Jones, N., et al. (2013). Nucleus-localized $21.5-\mathrm{kDa}$ myelin basic protein promotes oligodendrocyte proliferation and enhances neurite outgrowth in coculture, unlike the plasma membrane-associated 18.5 -kDa isoform. J. Neurosci. Res. 91, 349-362. doi: 10.1002/jnr.23166

Song, J., Carson, J. H., Barbarese, E., Li, F. Y., and Duncan, I. D. (2003). RNA transport in oligodendrocytes from the taiep mutant rat Mol. Cell. Neurosci. 24, 926-938. doi: 10.1016/S1044-7431(03)00254-9

Sperber, B. R., Boyle-Walsh, E. A. Engleka, M. J., Gadue, P., Peterson, A. C., Stein, P. L., et al. (2001). A unique role for Fyn in CNS myelination. J. Neurosci. 21, 2039-2047.

Staugaitis, S. M., Smith, P. R., and Colman, D. R. (1990). Expression of myelin basic protein isoforms in nonglial cells. J. Cell Biol. 110, 17191727. doi: $10.1083 /$ jcb.110.5.1719

Wake, H., Lee, P. R., and Fields, R. D. (2011). Control of local protein synthesis and initial events in myelination by action potentials. Science 333, 1647-1651. doi: 10.1126/science. 1206998

Wang, Y., Lacroix, G., Haines, J., Doukhanine, E., Almazan, G., and Richard, S. (2010). The QKI6 RNA binding protein localizes with the MBP mRNAs in stress granules of glial cells. PLOS ONE 5:e12824. doi: 10.1371/journal.pone. 0012824

White, R., Gonsior, C., Bauer, N. M., Kramer-Albers, E. M., Luhmann, H. J., and Trotter, J. (2012). Heterogeneous nuclear ribonucleoprotein (hnRNP) $\mathrm{F}$ is a novel component of oligodendroglial RNA transport granules contributing to regulation of myelin basic protein (MBP) synthesis. J. Biol. Chem. 287, 1742-1754. doi: 10.1074/jbc.M111.235010

White, R., Gonsior, C., Kramer-Albers, E. M., Stohr, N., Huttelmaier, S., and Trotter, J. (2008). Activation of oligodendroglial Fyn kinase enhances translation of mRNAs transported in hnRNP A2-dependent RNA granules. J. Cell Biol. 181, 579-586. doi: 10.1083/jcb.200706164

Zhang, Y., Lu, Z., Ku, L., Chen, Y., Wang, H., and Feng, Y. (2003). Tyrosine phosphorylation of QKI mediates developmental signals to regulate mRNA metabolism. EMBO J. 22, 1801-1810. doi: 10.1093/emboj/cdg171

Conflict of Interest Statement: The authors declare that the research was conducted in the absence of any commercial or financial relationships that could be construed as a potential conflict of interest.

Received: 30 July 2013; accepted: 10 September 2013; published online: 27 September 2013.

Citation: Müller C, Bauer NM, Schäfer I and White R (2013) Making myelin basic protein - from mRNA transport to localized translation. Front. Cell. Neurosci. 7:169. doi: 10.3389/fncel.2013.00169

This article was submitted to the journal Frontiers in Cellular Neuroscience.

Copyright (C) 2013 Müller, Bauer, Schäfer and White. This is an open-access article distributed under the terms of the Creative Commons Attribution License (CC BY). The use, distribution or reproduction in other forums is permitted, provided the original author(s) or licensor are credited and that the original publication in this journal is cited, in accordance with accepted academic practice. No use, distribution or reproduction is permitted which does not comply with these terms. 